\title{
An elementary approach to discrete models of dividend strategies
}

\author{
Hans U. Gerber, Elias S. W. Shiu ${ }^{\dagger}$ Hailiang Yang ${ }^{\ddagger}$
}

\begin{abstract}
The paper studies a discrete counterpart of Gerber et al (2006). The surplus of an insurance company (before dividends) is modeled as a time-homogeneous Markov chain with possible changes of size $+1,0,-1,-2,-3, \cdots$. If a barrier strategy is applied for paying dividends, it is shown that the dividends-penalty identity holds. The identity expresses the expected present value of a penalty at ruin in terms of the expected discounted dividends until ruin and the expected present value of the penalty at ruin if no dividends are paid. For the problem of maximizing the difference between the expected discounted dividends until ruin and the expected present value of the penalty at ruin, barrier strategies play a prominent role. In some cases an optimal dividend barrier exists. The paper discusses in detail the special case where the distribution of the change in
\end{abstract}

*Distinguished Visiting Professor at the University of Hong Kong, Ecole des hautes études commerciales, Université de Lausanne, CH-1015 Lausanne, Switzerland, e-mail: hgerber@unil.ch.

$\dagger$ Department of Statistics and Actuarial Science, The University of Iowa, Iowa City, Iowa 522421409, e-mail: eshiu@stat.uiowa.edu

$\ddagger$ Corresponding author, Department of Statistics and Actuarial Science, The University of Hong Kong, Pokfulam Road, Hong Kong, e-mail: hlyang@hkusua.hku.hk, telephone number: (852) 2857 8322, fax number: (852) 28589041. 
surplus does not depend on the current surplus (so that in the absence of dividends the surplus process has independent increments). A closed form result for zero initial surplus is given, and it is shown how the relevant quantities can be calculated recursively. Finally, it is shown how optimal dividend strategies can be determined; typically, they are band strategies.

Key words: Optimal dividends, penalty at ruin, dividends-penalty identity, band strategy, Lundberg equation.

JEL Classification: G22, G35.

Subject Categories: IM13, IM50.

\section{Introduction}

Traditionally, actuaries have been primarily concerned with the valuation of insurance and pension liabilities and the solvency of such financial systems. In the classical actuarial model for the probability of ruin, the surplus of an insurance company can increase without bounds. As this is unrealistic, de Finetti (1957) suggested that other considerations, such as dividend payments, should play a role. Specifically, he considered a discrete-time model, in which the periodic gains of a company are +1 or -1 . He sought the dividend-payment strategy that maximizes the expectation of the discounted dividends paid to the shareholders of the company. He found that such a dividend-payment strategy must be a barrier strategy, and showed how the optimal level of the barrier can be determined.

Within a few years after the publication of de Finetti's paper and apparently independent of it, several economists and operations researchers (Shubik and Thompson 1959, Miyasawa 1962, Takeuchi 1962) also became interested in the problem and some of its extensions. De Finetti's model has now been refined and generalized 
in many directions. Two excellent, recent surveys on dividend-payment strategies are Albrecher and Thonhauser (2009) and Avanzi (2009). Discussions on optimal dividend-payment strategies can be found in actuarial books such as Seal (1969), Bühlmann (1970), Borch (1974, 1990), Gerber (1979), Dickson (2005), and Schmidli (2008).

In this paper, we return to the doubly discrete framework of de Finetti. We assume that the surplus process is a time-homogeneous Markov chain. Following Gerber et al. (2006), we also assume that the surplus process is skip-free upward; in particular, we assume that only changes of size $+1,0,-1,-2,-3, \cdots$ from one period to the next are possible. (This is the case if the claims are multiples of the periodic premium; after a change of monetary units, the periodic premium is 1.) Then we show that the dividends-penalty identity of Gerber et al. (2006) also holds in this model. The identity expresses the expected present value of a penalty at ruin, if dividends are paid, in terms of the expected discounted dividends until ruin and the expected present value of the penalty at ruin, if no dividends are ever paid. Moreover, the dividends-penalty identity is crucial for the analysis of the optimal dividend barrier (which does not always exist).

The second half of the paper specializes to the case where the distribution of the change in surplus does not depend on the current surplus. (This is the discrete counterpart of the classical compound Poisson model.) It is shown that the expected discounted penalty at ruin, if no dividends are paid, can be calculated efficiently by recursion. A key quantity for the recursion algorithm is $A_{k}$, the expectation of the discounted value of a payment of 1 payable at the time of ruin, provided that the deficit at ruin is $k$. Several equivalent formulas for $A_{k}$ are derived. The roots of a version of the Lundberg fundamental equation play an important role. For zero initial surplus, there is a closed-form formula for the expected discounted penalty at ruin. 
The last section discusses the problem of maximizing the expected difference between the discounted dividends until ruin and the discounted penalty at ruin, if arbitrary dividend-payment strategies are admitted. The problem is a special case of discrete discounted dynamic programming. In general, the optimal strategy is a band strategy.

In comparison to the continuous model, the discrete model has several important merits; see also Li et al. (2009). The mathematics is more elementary and accessible to a broader readership. Recursive calculations are easy to implement. The discrete model can be used as an approximation to the continuous model, whereas the converse is not true. Finally, important concepts can be readily illustrated. For example, as we pointed out earlier, the optimal dividend-payment strategy in de Finetti's model is always a barrier strategy. However, if the periodic gains of the surplus process change from $\{+1,-1\}$ to $\{+1,-2\}$, then the optimal strategy is not necessarily a barrier strategy. Such an example, originally due to Morrill (1966), will be presented in Section 6 of the paper.

\section{Model}

The model is doubly discrete. The surplus of a company is considered only at times $0,1,2, \cdots$, and the surplus is assumed to be integer-valued at any time. It is assumed that the surplus is a time-homogeneous Markov chain, and that only changes of size $+1,0,-1,-2,-3, \cdots$ from one period to the next are possible. Ruin is the event that the surplus becomes negative sometime in the future.

For non-negative integers $u_{1} \leq u_{2}$, we define $C\left(u_{1}, u_{2}\right)$. Given an initial surplus

$u_{1}$, it is the expected present value (with respect to a constant interest rate) of a contingent payment of 1 at the time when the surplus reaches the level $u_{2}$, provided 
that ruin has not occurred in the meantime. If the surplus process is to go from 0 to $u_{2}$, it must pass through $u_{1}$; it follows from this and the time-homogeneity of the Markov chain that

$$
C\left(0, u_{2}\right)=C\left(0, u_{1}\right) C\left(u_{1}, u_{2}\right)
$$

Hence,

$$
C\left(u_{1}, u_{2}\right)=\frac{C\left(0, u_{2}\right)}{C\left(0, u_{1}\right)} .
$$

Thus, if one sets $h(u)=1 / C(0, u)$, or any positive multiple of that, we have

$$
C\left(u_{1}, u_{2}\right)=\frac{h\left(u_{1}\right)}{h\left(u_{2}\right)} .
$$

In the context of Lévy processes, the function $h(u)$ is known as a scale function.

\section{Dividends}

Until possible ruin, the company can pay dividends to its shareholders. As a consequence, the surplus is modified. We assume the transition probabilities are not affected, that is, they depend on the current value of the modified surplus.

We assume that dividends are paid according to a barrier strategy with positive parameter $b$, an integer. Whenever the surplus is above $b$, the excess is paid as a dividend. Let $V(u ; b)$ denote the expected present value of the dividends until ruin, if $u$ is the initial surplus. Obviously,

$$
V(u ; b)=u-b+V(b ; b), \quad u=b+1, b+2, \cdots .
$$

For $u \leq b$, the surplus must first reach the level $b+1$ before any dividends are paid. Hence,

$$
V(u ; b)=C(u, b+1) V(b+1 ; b)
$$


for $u=0,1, \cdots, b$. From this and (2.1) we gather that

$$
V(u ; b)=K(b) h(u)
$$

where $K(b)$ does not depend on $u$. Taking the forward difference with respect to $u$ and setting $u=b$ yields

$$
1=K(b)[h(b+1)-h(b)]=K(b) \Delta h(b) .
$$

It follows that

$$
V(u ; b)=\frac{h(u)}{\Delta h(b)}, \quad u=0,1, \cdots, b .
$$

This result is known as the factorization formula. Gerber (1972, formula 3.4) has derived (3.1) with the additional assumption that the probability distribution of the change in surplus does not depend on the current surplus. If the change in surplus can only be +1 and -1 and if the distribution does not depend on the current surplus, then (3.1) can be found in de Finetti (1957) and Shubik and Thompson (1959); Morrill (1966) has derived the result with -1 generalized to $-n$.

\section{The dividends-penalty identity}

We assume that at the time of ruin, a penalty is due. Such a payment is a given function of the deficit at ruin, and this function is the same for all dividend strategies.

The function $\phi(u)$ is defined as the expected discounted penalty at ruin, if no dividends are paid (the penalty is discounted with the same interest rate as the dividends). It is considered as a function of the initial surplus $u$. Similarly, $\phi(u ; b)$ denotes the expected discounted penalty at ruin, if dividends are paid according to the barrier strategy with parameter $b$. 
We shall prove the formula

$$
\phi(u ; b)=\phi(u)-\Delta \phi(b) \cdot V(u ; b), \quad u=0,1, \cdots, b,
$$

which is known as the dividends-penalty identity. It is a discrete counterpart of formula (9) in Gerber et al. (2006) which was obtained by a similar probabilistic reasoning. Important special cases of formula (9) in Gerber et al. (2006) are formulas (3.5) in Lin et al. (2003) and (3.3) in Yuen et al. (2007), which were obtained by analytical methods.

If the surplus does not reach the level $b+1$ before ruin, the penalties with and without dividends are the same. Hence,

$$
\begin{aligned}
\phi(u ; b)-\phi(u) & =C(u, b+1)[\phi(b+1 ; b)-\phi(b+1)] \\
& =h(u) \frac{\phi(b+1 ; b)-\phi(b+1)}{h(b+1)}
\end{aligned}
$$

by $(2.1)$. Obviously $\phi(b+1 ; b)=\phi(b ; b)$. So, if we take the forward difference of $(4.2)$ with respect to $u$ and set $u=b$, we see that

$$
-\Delta \phi(b)=\Delta h(b) \frac{\phi(b+1 ; b)-\phi(b+1)}{h(b+1)} .
$$

Combining (4.2) and (4.3), we obtain

$$
\phi(u ; b)-\phi(u)=-\Delta \phi(b) \frac{h(u)}{\Delta h(b)} .
$$

From this and (3.1), formula (4.1) follows.

\section{The optimal dividend barrier}

It is natural to choose $b$ which maximizes

$$
W(u ; b)=V(u ; b)-\phi(u ; b)
$$


A priori, it is not clear whether there is an optimal value of $b$ that is independent of $u$. For $u \leq b$, we have

$$
\begin{aligned}
W(u ; b) & =V(u ; b)-\phi(u)+\Delta \phi(b) V(u ; b) \\
& =\frac{1+\Delta \phi(b)}{\Delta h(b)} h(u)-\phi(u)
\end{aligned}
$$

by (4.1) and (3.1). Hence if $u \leq b$ the problem is to maximize the function

$$
\frac{1+\Delta \phi(b)}{\Delta h(b)}
$$

For $u<b$, we have

$$
W(u ; b+1)-W(u ; b)=h(u) \Delta\left\{\frac{1+\Delta \phi(b)}{\Delta h(b)}\right\}
$$

On the other hand, for $u \geq b$, we have

$$
\begin{aligned}
W(u ; b) & =u-b+W(b ; b) \\
& =u-b+h(b) \frac{1+\Delta \phi(b)}{\Delta h(b)}-\phi(b)
\end{aligned}
$$

by (5.2) with $u=b$. Applying the product rule for forward differences, we see after simplification that

$$
W(u ; b+1)-W(u ; b)=h(b+1) \Delta\left\{\frac{1+\Delta \phi(b)}{\Delta h(b)}\right\}
$$

for $u>b$. A comparison of (5.4) and (5.6) shows that the sign of $W(u ; b+1)-W(u ; b)$ does not depend on $u$. From this we gather that the local maxima of (5.2) and (5.5) are attained at the same values of $b$, that is, at the values of $b$ for which (5.3) attains a local maximum. Hence, in the case where there is only one maximum, there is an optimal dividend barrier $b^{*}$ that is independent of $u$. If there is more than one local maximum, it is possible that the global maximum of (5.2) and that of (5.5) are attained at different values of $b$. This will be illustrated by the examples in the next section. 


\section{Compound binomial model}

We consider the special case where the distribution of the change in surplus does not depend on the current surplus; in other words, in the absence of dividends, the surplus process would have independent and stationary increments. Let $p_{i}$ denote the probability for a one-period change in surplus of amount $i, i=+1,0,-1,-2, \cdots$ We assume that $p_{1}$, which is the probability of no claim in one period, is positive, and that $p_{1}+p_{0}<1$ (otherwise the problem would not be interesting). Let $v<1$ denote the one-period discount factor. By conditioning on the change in surplus in the first period, we see that

$$
V(u ; b)=v \sum_{i=-u}^{1} p_{i} V(u+i ; b)
$$

$u=0,1, \cdots, b-1$. Because of this and (3.1), and because $b$ is arbitrary, it follows that the scale function $h$ satisfies the equation

$$
h(u)=v \sum_{i=-u}^{1} p_{i} h(u+i),
$$

$u=0,1,2, \cdots$. Let $\Pi(x)$ denote the penalty that is due if the negative surplus at ruin is $x$. Then the function $\phi(u)$ satisfies the equation

$$
\phi(u)=v\left[\sum_{i=-u}^{1} p_{i} \phi(u+i)+\sum_{i=-\infty}^{-u-1} p_{i} \Pi(u+i)\right],
$$

$u=0,1,2, \cdots$

The functions $h$ and $\phi$ can be calculated recursively by rewriting (6.1) and (6.2) as

$$
h(u+1)=\frac{1}{p_{1}}\left[\frac{h(u)}{v}-\sum_{i=-u}^{0} p_{i} h(u+i)\right]
$$

and

$$
\phi(u+1)=\frac{1}{p_{1}}\left[\frac{\phi(u)}{v}-\sum_{i=-u}^{0} p_{i} \phi(u+i)-\sum_{i=-\infty}^{-u-1} p_{i} \Pi(u+i)\right]
$$


respectively. To apply (6.3), we note that $h(0)$ is an arbitrary positive constant and we set

$$
h(0)=1
$$

For applying (6.4), we need the value of $\phi(0)$. Fortunately, it has a closed-form expression. For initial surplus $u=0$, let $A_{k}$ denote the expectation of the discounted value of a payment of 1 payable at the time of ruin, provided that the deficit at ruin is $k$. The symbol $A_{k}$ is motivated by the international actuarial notation for net single premiums for insurances of unit benefit amount. Obviously,

$$
\phi(0)=\sum_{k=1}^{\infty} A_{k} \Pi(-k) .
$$

We shall show in Section 7 that

$$
A_{k}=\frac{r_{0}^{k-1}}{p_{1}} \sum_{i=-\infty}^{-k} p_{i} r_{0}^{i}, \quad k=1,2,3, \cdots
$$

Here, $r_{0}$ is a particular solution of the following equation for $r$,

$$
v \sum_{i=-\infty}^{1} p_{i} r^{i}=1
$$

it is the solution greater than $1 / v$ (To see the existence and uniqueness of $r_{0}$, note that the expression on the left hand side of (6.8) is less than 1 for $r=1 / v$, tends to $\infty$ for $r \rightarrow \infty$, and has a positive second derivative.) Equation (6.8) is a form of Lundberg's Fundamental Equation. We note that formula (6.7) is essentially formula (5.1) in Cheng et al. (2000), where ruin is defined slightly differently; both formulas can be viewed as discrete counterparts of formula (2.30) in Gerber and Shiu (1998),

$$
g(x)=\frac{\lambda}{c} \int_{x}^{\infty} e^{-\rho(y-x)} p(y) d y
$$

where $e^{\rho}$ plays the role of $r_{0}$. Formula (6.7) can be obtained from the last formula on page 6 of $\mathrm{Li}$ and Garrido (2002) and also from $m_{*}(0)$ on page 273 of Pavlova and Willmot (2004). Section 7 will present an independent and more accessible proof which seems to have merits on its own. 
The recursive methods are easy to implement. We consider three examples.

Example 1. Suppose $p_{1}=0.5, p_{0}=0.2, p_{-1}=0.2$ and $p_{-2}=0.1$. We consider three different discount factors and five penalty functions of the form $\Pi(x)=K|x|$. In all fifteen cases, it is found that expression (5.3) has a unique maximum. Hence $W(u ; b)$ is maximized at $b=b^{*}$, independently of $u$. The optimal dividend barriers $b^{*}$ are shown in Table 1 . We note that $b^{*}$ is an increasing function of both $K$ and the discount factor $v$.

Table 1: The influence of $v$ and $\Pi$ on $b^{*}$

\begin{tabular}{|c|c|c|c|c|c|}
\hline$v$ & $K=0$ & $K=1$ & $K=2$ & $K=5$ & $K=10$ \\
\hline 0.95 & 0 & 1 & 2 & 3 & 5 \\
\hline 0.99 & 6 & 7 & 7 & 8 & 10 \\
\hline 0.999 & 29 & 29 & 29 & 29 & 30 \\
\hline
\end{tabular}

Example 2. Morrill's (1966) example 2 is for $p_{1}=12 / 13, p_{-2}=1 / 13$ and $v=65 / 72$. There is no penalty at ruin. According to $(6.3), h$ can be calculated recursively by the formula

$$
h(u+1)=\frac{6}{5} h(u)-\frac{1}{12} h(u-2),
$$

$u=0,1,2, \cdots$. It is found that expression (5.3) with $\phi=0$ has two local maxima, at $b=0$ and $b=2$. A glance at Table 2 shows that for $u=0,1$ the expression $V(u ; b)$ is maximized by $b=0$ and that for all other values of $u, V(u ; b)$ is maximized by $b=2$. We consider now the same example, but with a penalty function $\Pi(x)=|x|$. Then $\phi$ is determined recursively by the formula

$$
\phi(u+1)=\frac{6}{5} \phi(u)-\frac{1}{12} \phi(u-2)
$$

$u=0,1,2, \cdots$. Here, $\phi(0)=A_{1}+2 A_{2}$ with

$$
A_{1}=\frac{1}{12} r_{0}^{-2}, \quad A_{2}=\frac{1}{12} r_{0}^{-1},
$$


where $r_{0}=1.13535$. It is found that (5.3) has two local maxima, at $b=0$ and $b=2$. However, Table 3 shows that $W(u ; b)$ is maximized by $b=2$ for any value of $u$. Hence $b^{*}=2$ is the optimal dividend barrier.

Table 2: The values of $V(u ; b)$

\begin{tabular}{|c|c|c|c|c|c|c|c|c|}
\hline & $b=0$ & $b=1$ & $b=2$ & $b=3$ & $b=4$ & $b=5$ & $b=6$ & $b=7$ \\
\hline$u=0$ & 5 & 4.167 & 4.886 & 4.368 & 3.926 & 3.465 & 3.056 & 2.692 \\
\hline$u=1$ & 6 & 5 & 5.863 & 5.242 & 4.711 & 4.158 & 3.667 & 3.230 \\
\hline$u=2$ & 7 & 6 & 7.036 & 6.290 & 5.653 & 4.990 & 4.400 & 3.876 \\
\hline$u=3$ & 8 & 7 & 8.036 & 7.184 & 6.457 & 5.699 & 5.026 & 4.427 \\
\hline
\end{tabular}

Table 3: The values of $W(u ; b)$ when $\Pi(x)=|x|$

\begin{tabular}{|l|l|l|l|l|l|l|l|l|}
\hline & $b=0$ & $b=1$ & $b=2$ & $b=3$ & $b=4$ & $b=5$ & $b=6$ & $b=7$ \\
\hline$u=0$ & 4.167 & 3.681 & 4.609 & 4.132 & 3.709 & 3.252 & 2.844 & 2.480 \\
\hline$u=1$ & 5.167 & 4.583 & 5.698 & 5.125 & 4.618 & 4.069 & 3.579 & 3.143 \\
\hline$u=2$ & 6.167 & 5.583 & 6.921 & 6.233 & 5.625 & 4.966 & 4.379 & 3.855 \\
\hline$u=3$ & 7.167 & 6.583 & 7.921 & 7.135 & 6.440 & 5.688 & 5.017 & 4.419 \\
\hline
\end{tabular}

Example 3. Suppose $p_{1}=0.75, p_{0}=0.05, p_{-1}=0.1$ and $p_{-6}=0.1, v=0.999$, and no penalty at ruin. It is found that expression (5.3) with $\phi=0$ has three local maxima, at $b=1,7,38$. In Table 4 , the values of $V(0 ; b), V(1 ; b)$ and $V(50 ; b)$ around the local maxima are exhibited. Thus $V(0 ; b)$ and $V(1 ; b)$ are maximized at $b=1$, but $V(50 ; b)$ is maximized at $b=38$. Hence, in this example, an optimal dividend barrier does not exist and the optimal optimal dividend strategy cannot be a barrier strategy. 
Table 4: The values of $V(u ; b)$

\begin{tabular}{|c|c|c|c|c|c|c|c|c|}
\hline$u$ & $b=1$ & $b=2$ & $b=6$ & $b=7$ & $b=8$ & $b=37$ & $b=38$ & $b=39$ \\
\hline 0 & 4.8428 & 4.4228 & 3.8031 & 3.9710 & 3.8952 & 4.7536 & 4.7556 & 4.7555 \\
\hline 1 & 6.1407 & 5.6082 & 4.8223 & 5.0352 & 4.9391 & 6.0276 & 6.0302 & 6.0301 \\
\hline 50 & 55.1407 & 54.5214 & 53.8961 & 54.3772 & 54.1410 & 59.4013 & 59.4212 & 59.4204 \\
\hline
\end{tabular}

Remark 1. In view of (6.8), an alternative expression for (6.7) is

$$
A_{k}=\frac{r_{0}^{k-1}}{p_{1}}\left(\frac{1}{v}-\sum_{i=-k+1}^{1} p_{i} r_{0}^{i}\right), \quad k=1,2,3, \cdots .
$$

From (6.10) or (6.7) it follows that

$$
A_{k+1}=r_{0} A_{k}-\frac{p_{-k}}{p_{1}}, \quad k=1,2,3, \cdots
$$

Thus the $A_{k}$ 's can be calculated recursively, starting with

$$
A_{1}=\frac{1}{p_{1}}\left(\frac{1}{v}-p_{0}-p_{1} r_{0}\right)
$$

Remark 2. By conditioning on the first time when the surplus falls below the initial surplus and on the amount by which it falls below, we see that

$$
\phi(u)=\sum_{k=1}^{u} \phi(u-k) A_{k}+\sum_{k=u+1}^{\infty} \Pi(u-k) A_{k}, \quad u=1,2,3, \cdots .
$$

This equation (which is a discrete renewal equation) provides another recursive method, alternative to (6.4), for calculating $\phi(u)$. Both methods start with (6.6). Equation (6.13) is a discrete counterpart of (2.34) in Gerber and Shiu (1998).

Remark 3. Information about the asymptotic behavior of the function $\phi(u)$ can be obtained as follows. The second derivative of the expression on the lefthand side of (6.8) is positive for $r>0$. It follows that equation (6.8) has at most two positive solutions. One is $r_{0}$. Under some mild technical conditions on the 
asymptotic behavior of the $p_{k}$ 's, equation (6.8) has another positive solution $r_{1}<1$. With a martingale argument that will be explained in Remark 8 in the next section, one can show that

$$
\sum_{k=1}^{\infty} r_{1}^{-k} A_{k}=1
$$

(See also (7.11) in the next section.) Hence, the defective renewal equation (6.13) for the function $\phi(u)$ can be turned into a proper renewal equation for the function $\phi(u) r_{1}^{-u}$. From this, we obtain the asymptotic result that $\phi(u) r_{1}^{-u}$ tends to a positive constant for $u \rightarrow \infty$.

Remark 4. Let $I$ denote the identity operator and $E$ the forward shift operator. Then, (6.7) is

$$
\begin{aligned}
A_{k} & =\frac{1}{p_{1} r_{0}} \sum_{j=0}^{\infty} r_{0}^{-j} p_{-k-j} \\
& =\frac{1}{p_{1} r_{0}} \sum_{j=0}^{\infty} r_{0}^{-j} E^{-j} p_{-k} \\
& =\frac{1}{p_{1}}\left(r_{0} I-E^{-1}\right)^{-1} p_{-k}
\end{aligned}
$$

since $r_{0}>1$. In functional analysis, for a given linear operator $S$, the function

$$
R(z ; S)=(z I-S)^{-1}
$$

is called the resolvent function of $S$, or simply, the resolvent of $S$. Thus,

$$
A_{k}=\frac{1}{p_{1}} R\left(r_{0} ; E^{-1}\right) p_{-k}
$$

It has been pointed out in Gerber and Shiu (2005) that (6.9) can be expressed as

$$
g(x)=\frac{\lambda}{c} R(\rho ; D) p(x)
$$

where $D$ is the differentiation operator, and that the operator $T_{\rho}$ in Dickson and Hipp (2001) is the resolvent $R(\rho ; D)$. In Albrecher et al. (2010), $R(\rho ; D)$ is denoted as $B_{\rho}$. 
The operator $T_{r}$ in $\mathrm{Li}(2005)$ and $\mathrm{Li}$ et al. (2009) is $R(1 ; r E)$. A paper related to the Dickson-Hipp operator is Redheffer (1966).

Remark 5. In some cases, we can evaluate (6.14) by the method of power shift (Brand, 1966, Section 90). For example, suppose that

$$
p_{-k}=\left(1-p_{1}\right) f_{N B}(k), \quad k=0,1,2, \cdots,
$$

where

$$
f_{N B}(k)=\left(\begin{array}{c}
n-1+k \\
n-1
\end{array}\right)(1-\theta)^{n} \theta^{k}, \quad k=0,1,2, \cdots
$$

with $n$ being a fixed positive integer. Then, (6.14) is

$$
A_{k}=\frac{1-p_{1}}{p_{1}}\left(r_{0} I-E\right)^{-1} f_{N B}(k), \quad k=1,2,3, \cdots
$$

By the power shift formula,

$$
h(E)\left[g(k) \theta^{k}\right]=\theta^{k} h(\theta E) g(k),
$$

we have

$$
\begin{aligned}
& \left(r_{0} I-E\right)^{-1}\left[\left(\begin{array}{c}
n-1+k \\
n-1
\end{array}\right) \theta^{k}\right] \\
= & \theta^{k}\left(r_{0} I-\theta E\right)^{-1}\left(\begin{array}{c}
n-1+k \\
n-1
\end{array}\right) \\
= & \frac{\theta^{k}}{r_{0}-\theta}\left(I-\frac{\theta}{r_{0}-\theta} \Delta\right)^{-1}\left(\begin{array}{c}
n-1+k \\
n-1
\end{array}\right) \\
= & \frac{\theta^{k}}{r_{0}-\theta}\left[I+\frac{\theta}{r_{0}-\theta} \Delta+\left(\frac{\theta}{r_{0}-\theta}\right)^{2} \Delta^{2}+\cdots\right]\left(\begin{array}{c}
n-1+k \\
n-1
\end{array}\right) \\
= & \theta^{k-1} \sum_{j=1}^{n}\left(\frac{\theta}{r_{0}-\theta}\right)^{j}\left(\begin{array}{c}
n-1+k \\
n-j
\end{array}\right) .
\end{aligned}
$$


It follows from (6.17), (6.16) and (6.18) that

$$
A_{k}=\frac{1-p_{1}}{p_{1}}(1-\theta)^{n} \theta^{k-1} \sum_{j=1}^{n}\left(\frac{\theta}{r_{0}-\theta}\right)^{j}\left(\begin{array}{c}
n-1+k \\
n-j
\end{array}\right), \quad k=1,2,3, \cdots .
$$

By linearity, this method to evaluate $A_{k}$ remains applicable even if the function $f_{N B}$ in (6.15) is replaced by a linear combination of such functions.

\section{$7 \quad$ Formula (6.7)}

In this section, we assume that only changes of the surplus of $+1,0,1,2, \cdots,-m$ are possible. That is, the largest possible negative change of the surplus in each period is $m$ (thus $m=2$ in Examples 1 and 2, and $m=6$ in Example 3). Clearly, it suffices to prove (6.7) under this assumption. Also, in this special case, the functions $h(u)$ and $\phi(u)$ can be obtained in a transparent fashion. First, it is judicious to extend their domain of definition to $u=-1,-2, \cdots,-m$ by setting

$$
h(u)=0 \quad \text { for } u=-1,-2, \cdots,-m,
$$

and

$$
\phi(u)=\Pi(u) \quad \text { for } u=-1,-2, \cdots,-m .
$$

Then, equations (6.1) and (6.2) can be written as

$$
h(u)=v \sum_{j=-m}^{1} p_{j} h(u+j)
$$

and

$$
\phi(u)=v \sum_{j=-m}^{1} p_{j} \phi(u+j),
$$

for $u=0,1,2, \cdots$. The characteristic polynomial of these homogeneous difference equations is

$$
P(r)=r^{m}-v \sum_{j=-m}^{1} p_{j} r^{m+j}
$$


Because $\left|P(r)-r^{m}\right|<1$ for $|r|=1$, it follows from Rouché's Theorem that $P(r)$ has exactly $m$ zeros, $r_{1}, r_{2}, \cdots, r_{m}$, inside the unit circle. Typically, the zeros are distinct, and we make this assumption temporarily. Also, note that the remaining zero $r_{0}>1 / v$ has already been introduced in Section 6 . Now, the general solutions of (7.3) and (7.4) are of the form

$$
h(u)=\sum_{k=0}^{m} C_{k} r_{k}^{u}
$$

and

$$
\phi(u)=\sum_{k=0}^{m} D_{k} r_{k}^{u}
$$

The boundary conditions (6.5) and (7.1) yield a system of $m+1$ linear equations for the coefficients $C_{0}, C_{1}, \cdots, C_{m}$ :

$$
\begin{aligned}
& C_{0}+C_{1}+\cdots+C_{m}=1, \\
& \sum_{k=0}^{m} C_{k} r_{k}^{x}=0, \quad x=-1,-2, \cdots,-m .
\end{aligned}
$$

As for the function $\phi(u)$, we note that $D_{0}=0$ because $\phi(u) \rightarrow 0$ for $u \rightarrow \infty$. The boundary conditions (7.2) yield a system of $m$ linear equations

$$
\sum_{k=1}^{m} D_{k} r_{k}^{x}=\Pi(x), \quad x=-1,-2, \cdots,-m
$$

from which $D_{1}, D_{2}, \cdots, D_{m}$ can be determined. The coefficient matrices of both (7.7) and (7.8) are Vandermonde matrices and therefore invertible as the $r_{k}$ 's are assumed to be distinct. Examples solving (7.8) with $v=1$ can be found in Liu and Guo (2006).

Our next goal is to show that the $A_{k}$ 's satisfy a system of $m$ linear equations. For this, it is convenient to use matrix notation. Let $\mathbf{R}$ be the $m \times m$ matrix with element $r_{k}^{-j}$ in row $k$ and column $j$, and let

$$
\mathbf{D}=\left(D_{1}, D_{2}, \cdots, D_{m}\right)
$$


and

$$
\Pi=(\Pi(-1), \Pi(-2), \cdots, \Pi(-m))
$$

be two $m$-dimensional row vectors. Then, (7.8) states that

$$
\mathrm{DR}=\Pi,
$$

or

$$
\mathrm{D}=\Pi \mathrm{R}^{-1}
$$

Because of (7.6),

$$
\phi(0)=\sum_{k=1}^{m} D_{k}=\mathbf{D} \mathbf{1}=\mathbf{\Pi} \mathbf{R}^{-\mathbf{1}} \mathbf{1}
$$

where $\mathbf{1}$ is the $m$-dimensional column vector with all entries being 1 . From (7.9) and (6.6), we see that

$$
\mathbf{R}^{-1} \mathbf{1}=\mathbf{A}
$$

where

$$
\mathbf{A}=\left(A_{1}, A_{2}, \cdots, A_{m}\right)^{T}
$$

It follows from (7.10) that

$$
\mathbf{R A}=\mathbf{1}
$$

or

$$
\sum_{k=1}^{m} r_{j}^{-k} A_{k}=1, \quad j=1,2, \cdots, m
$$

This is a system of $m$ linear equations for determining $A_{1}, A_{2}, \cdots, A_{m}$.

To derive (6.7), consider the generating function

$$
G(y)=\sum_{k=1}^{m} A_{k} y^{k}
$$


Then, (7.11) means that

$$
G\left(1 / r_{n}\right)=1, \quad n=1,2, \cdots, m
$$

Since the polynomial $G(y)$ vanishes at $y=0$, it can be written as

$$
G(y)=1-\prod_{n=1}^{m}\left(1-r_{n} y\right)
$$

Because $r_{0}, r_{1}, \cdots, r_{m}$ are the zeros of $P(r)$, we have

$$
\begin{aligned}
P(r) & =-v p_{1}\left(r-r_{0}\right) \prod_{n=1}^{m}\left(r-r_{n}\right) \\
& =-v p_{1}\left(r-r_{0}\right) r^{m}[1-G(1 / r)] \\
& =-v p_{1}\left(r-r_{0}\right)\left(r^{m}-\sum_{k=1}^{m} A_{k} r^{m-k}\right) \\
& =-v p_{1}\left[r^{m+1}-\left(r_{0}+A_{1}\right) r^{m}+\sum_{k=1}^{m-1}\left(r_{0} A_{k}-A_{k+1}\right) r^{m-k}+r_{0} A_{m}\right]
\end{aligned}
$$

Comparing (7.15) with $(7.5)$ yields $(m+1)$ equations:

$$
\begin{aligned}
1-v p_{0} & =v p_{1}\left(r_{0}+A_{1}\right), \\
v p_{-k} & =v p_{1}\left(r_{0} A_{k}-A_{k+1}\right), \quad k=1,2, \cdots, m-1, \\
v p_{-m} & =v p_{1} r_{0} A_{m} .
\end{aligned}
$$

Note that (7.16) and (7.17) are equivalent to (6.12) and (6.11), respectively. Clearly, these equations hold even if some of the $r_{k}$ 's coincide. To solve for the $A_{k}$ 's, we rewrite formulas (7.17) and (7.18) as

$$
\begin{aligned}
r_{0}^{-(j+1)} p_{-j} & =p_{1}\left(r_{0}^{-j} A_{j}-r_{0}^{-(j+1)} A_{j+1}\right), \quad j=1,2, \cdots, m-1, \\
r_{0}^{-(m+1)} p_{-m} & =p_{1} r_{0}^{-m} A_{m} .
\end{aligned}
$$

By summing (7.19) from $j=k$ to $j=m-1$, adding the resulting equation to (7.20), and changing $j$ to $-j$, we obtain

$$
\sum_{j=-m}^{-k} r_{0}^{j-1} p_{j}=p_{1} r_{0}^{-k} A_{k}, \quad k=1,2, \cdots, m
$$


from which (6.7) follows.

Remark 6. In deriving (7.21), we did not use (7.16), which can be rewritten as

$$
-r_{0}^{-1} \frac{1}{v}+p_{1}+r_{0}^{-1} p_{0}=-p_{1} r_{0}^{-1} A_{1} \text {. }
$$

By summing (7.19) from $j=1$ to $j=k-1$ and adding the resulting equation to (7.22), we obtain

$$
-r_{0}^{-1} \frac{1}{v}+\sum_{j=-1}^{k-1} r_{0}^{-(j+1)} p_{-j}=-p_{1} r_{0}^{-k} A_{k}, \quad k=1,2,3, \cdots
$$

which is equivalent to (6.10).

Remark 7. There is a closed-form solution of the system of equations (7.11):

$$
A_{k}=(-1)^{k-1} \sigma_{k}\left(r_{1}, r_{2}, \cdots, r_{m}\right)
$$

where $\sigma_{k}\left(r_{1}, r_{2}, \cdots, r_{m}\right)$ is the elementary symmetric polynomial of degree $k$ in $r_{1}$, $r_{2}, \cdots, r_{m}$. This result is obtained by expanding the product in (7.14) and then comparing with (7.12). Hence it holds for arbitrary $r_{k}$ 's.

Remark 8. An alternative proof of (7.11) uses martingales. Consider a stochastic process of the form $\left\{v^{t} r^{U(t)} ; t \geq 0\right\}$, where $U(t)$ is the surplus (in the model without dividends) at time $t$. This process is a martingale, provided that $r$ is a zero of $P(r)$. In particular, for $j=1,2, \cdots, m$, the process $\left\{v^{t} r_{j}^{U(t)} ; 0 \leq t<T\right\}$ is a bounded martingale, where $T$ is the time of ruin. If we start at $U(0)=0$, stop it at time $T$, and use the optional sampling theorem, we obtain (7.11).

Remark 9. Applying (7.2) to (6.13) yields

$$
\phi(u)=\sum_{k=1}^{m} \phi(u-k) A_{k},
$$

which should be compared with (7.4). With the function $G$ defined by (7.12), the characteristic equation of $(7.23)$ is

$$
1=G(1 / r)
$$


Thus, $\phi(u)$ is a linear combination of the $m$ roots of (7.24), each raised to the power $u$. To reconcile this result with (7.6), we can use (7.13).

\section{Remarks on optimal dividend strategies}

So far, our discussion focussed on barrier strategies. We now consider the more general problem of maximizing the expected difference between the discounted dividends until ruin and the discounted penalty at ruin, if arbitrary dividend strategies are admitted. For each initial surplus $u \geq 0$, let $W(u)$ denote the maximal value of the expected difference. A dividend strategy is optimal, if the resulting expected difference is $W(u)$ for each $u \geq 0$. The problem of finding $W(u)$ and an optimal dividend strategy is a special case of discrete discounted dynamic programming. For an excellent introduction to this topic, the reader is referred to the first three sections of Blackwell (1962). In particular, it is shown that the search for optimal strategies can be limited to stationary strategies, that is, strategies where the decision at any time depends only on the state of the system at that time.

If the initial surplus is $u>0$, an option is to pay an immediate dividend of 1 and to continue with a new initial surplus of $u-1$. It follows that $W(u)$ is at least $W(u-1)+1$. If the function $W(u)$ is known, an optimal dividend strategy is readily obtained as follows: Let $u$ be the current surplus, no dividend is paid if $W(u)>W(u-1)+1$, and a dividend of amount

$$
\max \{d \mid W(u-d)=W(u)-d\}
$$

is paid if $W(u)=W(u-1)+1$. Such an optimal strategy is a band strategy. In some cases, it is reduced to a barrier strategy.

The maximal value function, $W(u)$, can be obtained by Bellman's recursive algo- 
rithm. To explain it, we shall again assume the compound binomial model that was considered in Section 6. We choose a function $w(u)$, set $W_{1}(u)=w(u)$, and calculate $W_{2}(u), W_{3}(u), \cdots$ recursively according to the formula

$$
W_{n+1}(u)=\max _{d=0,1, \cdots, u}\left\{d+v\left[\sum_{j=d-u}^{1} p_{j} W_{n}(u-d+j)-\sum_{j=-\infty}^{d-u-1} p_{j} \Pi(u-d+j)\right]\right\} .
$$

Note that $W_{n}(u)$ can be interpreted as the maximal expected difference of an $n$-period "game", with a terminal payoff of $w(x)$ and $x$ being the terminal surplus, if ruin has not occurred by time $n$. Under fairly general conditions for the function $w(u), W_{n}(u)$ converges to $W(u)$ for each $u$. Then, (8.2) becomes a functional equation for $W(u)$,

$$
W(u)=\max _{d=0,1, \cdots, u}\left\{d+v\left[\sum_{j=d-u}^{1} p_{j} W(u-d+j)-\sum_{j=-\infty}^{d-u-1} p_{j} \Pi(u-d+j)\right]\right\} .
$$

This is a discrete counterpart of the Hamilton-Jacobi-Bellman equation.

If $W_{n+1}(u-1)$ is already known, we have to consider only $d=0$ and 1 in (8.2). Thus

$$
W_{n+1}(u)=\max \left\{v\left[\sum_{j=-u}^{1} p_{j} W_{n}(u+j)-\sum_{j=-\infty}^{-u-1} p_{j} \Pi(u+j)\right], 1+W_{n+1}(u-1)\right\}
$$

for $u=1,2,3, \cdots$. Thus (8.4) provides a doubly recursive method, starting with

$$
W_{n+1}(0)=v\left[p_{0} W_{n}(0)+p_{1} W_{n}(1)\right] .
$$

In the limit, (8.4) gives rises to the equation

$$
W(u)=\max \left\{v\left[\sum_{j=-u}^{1} p_{j} W(u+j)-\sum_{j=-\infty}^{-u-1} p_{j} \Pi(u+j)\right], 1+W(u-1)\right\},
$$

which is an alternative to (8.3).

When is there an optimal dividend strategy that is a barrier strategy? Clearly, a necessary condition is the existence of an optimal dividend barrier $b^{*}$ which was 
discussed in Section 5. However, we believe that in general this condition is not sufficient, and that $W\left(u ; b^{*}\right)=W(u)$ has to be verified in each case. For this purpose, one sets $W_{1}(u)=W\left(u ; b^{*}\right)$ and verifies that $(8.2)$ leads to $W_{2}(u)=W\left(u ; b^{*}\right)$. In other words, one verifies that $W\left(u ; b^{*}\right)$ is a solution of (8.3). This condition has been verified in each of the 15 cases considered in Example 1. Thus the 15 barrier strategies with respective parameter $b^{*}$ are indeed optimal dividend strategies.

In the following, the maximal value function is denoted as $V(u)$ in the special case when $\Pi=0$.

Example 4. For the model and assumptions of Example 2, Bellman's recursive algorithm was applied to obtain the maximal value functions $V$ and $W$ as limits. The results are displayed in Table 5. From this and (8.1), the optimal dividend strategies can be deduced. In the absence of a penalty, the optimal dividend strategy is the following band strategy: Pay a dividend of 1 if $u=1$, and $u-2$ if $u>2$, and no dividend if $u=0$ or 2 . But, with the penalty at ruin, the barrier strategy with parameter $b^{*}=2$ is optimal.

Table 5: The values of $V(u)$ and $W(u)$ when $\Pi(x)=|x|$

\begin{tabular}{|c|c|c|c|c|c|c|c|c|}
\hline$u$ & 0 & 1 & 2 & 3 & 4 & 5 & 6 & 7 \\
\hline$V(u)$ & 5 & 6 & 7.083 & 8.083 & 9.083 & 10.083 & 11.083 & 12.083 \\
\hline$W(u)$ & 4.609 & 5.698 & 6.921 & 7.921 & 8.921 & 9.921 & 10.921 & 11.921 \\
\hline
\end{tabular}

Example 5. For the model and assumptions of Example 3, Bellman's recursive algorithm was applied to obtain the maximal value function as a limit. Table 6 shows the values of $V(u)$ for $u=0,1, \cdots, 39$. For $u \geq 39, V(u)=V(38)+u-38$. We also notice that $V(2)=V(1)+1$, and that in all other cases $V(u+1)>V(u)+1$. According to (8.1), the optimal band strategy is as follows: if the current surplus is 2 , a dividend of 1 is paid. If it is $u>38$, a dividend of $u-38$ is paid. For any other value 
of $u$, no dividend is paid. Comparing Tables 4 and 6 , we see that $V(u ; 1)=V(u)$ for $u=0,1$ and that $V(50 ; 38)=59.4212$ is less than $V(50)=V(38)+12=59.4784$.

Table 6: The values of $V(u)$

\begin{tabular}{|c|c|c|c|c|c|c|c|c|}
\hline$u$ & 0 & 1 & 2 & 3 & 4 & 5 & 6 & 7 \\
\hline$V(u)$ & 4.8428 & 6.1407 & 7.1407 & 8.1487 & 9.3805 & 10.8079 & 12.4538 & 13.7046 \\
\hline$u$ & 8 & 9 & 10 & 11 & 12 & 13 & 14 & 15 \\
\hline$V(u)$ & 14.8982 & 16.1116 & 17.3566 & 18.6093 & 19.8413 & 21.0170 & 22.1769 & 23.3316 \\
\hline$u$ & 16 & 17 & 18 & 19 & 20 & 21 & 22 & 23 \\
\hline$V(u)$ & 24.4793 & 25.6148 & 26.7344 & 27.8384 & 28.9323 & 30.0175 & 31.0937 & 32.1606 \\
\hline$u$ & 24 & 25 & 26 & 27 & 28 & 29 & 30 & 31 \\
\hline$V(u)$ & 33.2186 & 34.2686 & 35.3116 & 36.3484 & 37.3793 & 38.4047 & 39.4252 & 40.4415 \\
\hline$u$ & 32 & 33 & 34 & 35 & 36 & 37 & 38 & 39 \\
\hline$V(u)$ & 41.4540 & 42.4633 & 43.4699 & 44.4742 & 45.4767 & 46.4780 & 47.4784 & 48.4784 \\
\hline
\end{tabular}

Remark 10. If $W_{n}(u)$ is known for $u=0,1, \cdots, m$, the recursive formula (8.2) produces the values of $W_{n+1}(u)$ for $u=0,1, \cdots, m-1$ only. Thus with each iteration, the last component disappears. This poses a problem, if the convergence is slow, which necessitates a large number of iterations. The problem can be solved with the following technique. Choose $m$ large enough so that we can be sure that $W(u)=$ $W(u-1)+1$, for all $u \geq m$. Then apply (8.2) for $u=0,1, \cdots, m-1$ and set $W_{n+1}(m)=W_{n+1}(m-1)+1$.

Acknowledgements. The authors wish to thank two anonymous referees; their comments led to several improvements in the paper. Elias Shiu gratefully acknowledges the generous support from the Principal Financial Group Foundation. Hailiang Yang would like to acknowledge the Research Grants Council of the Hong Kong Spe- 
cial Administrative Region, China (project No. HKU 754008H).

\section{References}

Albrecher, H., Constantinescu, C., Pirsic, G., Regensburger, G., Rosenkranz, M., 2010. An algebraic operator approach to the analysis of Gerber-Shiu functions. Insurance: Mathematics and Economics, to appear.

Albrecher, H., Thonhauser, S., 2009. Optimality results for dividend problems in insurance. RACSAM Revista de la Real Academia de Ciencias; Serie A, Matemáticas 103 (2), 295-320.

Avanzi, B., 2009. Strategies for dividend distribution: a review. North American Actuarial Journal 13, 217-251.

Blackwell, D., 1962. Discrete dynamic programming. Annals of Mathematical Statistics 33 (2), 719-726.

Borch, K., 1974. The Mathematical Theory of Insurance. Lexington Books, Lexington, MA.

Borch, K., 1990. Economics of Insurance. North-Holland, Amsterdam.

Brand, L., 1966. Differential and Difference Equations. Wiley, New York.

Bühlmann, H., 1970. Mathematical Methods in Risk Theory. Springer-Verlag, Heidelberg.

Cheng, S., Gerber, H.U., Shiu, E.S.W., 2000. Discounted probabilities and ruin theory in the compound binomial model. Insurance: Mathematics and Economics 26, 239-250. 
de Finetti, B., 1957. Su un'impostazione alternativa della teoria collettiva del rischio. Transactions of the XVth International Congress of Actuaries 2, 433-443.

Dickson, D.C.M., 2005. Insurance Risk and Ruin. International Series on Actuarial Science, Cambridge University Press, Cambridge.

Dickson, D.C.M., Hipp, C., 2001. On the time to ruin for Erlang(2) risk processes. Insurance: Mathematics and Economics 29, 333-344.

Gerber, H.U., 1972. Games of economic survival with discrete- and continuousincome processes. Operations Research 20 (1), 37-45.

Gerber, H. U., 1979. An Introduction to Mathematical Risk Theory. S.S. Huebner Foundation Monograph Series No. 8. Irwin, Homewood, IL.

Gerber, H.U., Shiu, E.S.W., 1998. On the time value of ruin. North American Actuarial Journal 2 (1), 48-78.

Gerber, H.U., Shiu, E.S.W., 2005. The time value of ruin in a Sparre Andersen model. North American Actuarial Journal 9 (2), 49-84.

Gerber, H.U., Lin, X. S., Yang, H., 2006. A note on the dividends-penalty identity and the optimal dividend barrier. ASTIN Bulletin 36 (2), 489-503.

Li, S., 2005. On a class of discrete time renewal risk models. Scandinavian Actuarial Journal 2005 (4), 241-260.

Li, S., Garrido, J., 2002. On the time value of ruin in the discrete time risk model. Working paper 02-18, Business Economics, Universidad Carlos III de Madrid.

Li, S., Lu, Y., Garrido, J., 2009. A review of discrete-time risk models. RACSAM Revista de la Real Academia de Ciencias; Serie A, Matemáticas 103 (2), 321337. 
Lin, X.S., Willmot, G.E., Drekic, S., 2003. The classical risk model with a constant dividend barrier: analysis of the Gerber-Shiu discounted penalty function. Insurance: Mathematics and Economics 33, 551-566.

Liu, S.X., Guo, J.Y., 2006. Discrete risk model revisited. Methodology and Computing in Applied Probability 8, 303-313.

Miyasawa, K., 1962. An economic survival game. Journal of Operations Research Society of Japan 4, 95-113.

Morrill, J.E., 1966. One-person games of economic survival. Naval Research Logistics Quarterly 13, 49-69.

Pavlova, K.P., Willmot, G.E., 2004. The discrete stationary renewal risk model and the Gerber-Shiu discounted penalty function. Insurance: Mathematics and Economics 35, 267-277.

Redheffer, R., 1966. Algebraic properties of certain integral transforms. The American Mathematical Monthly, 73 (4), Part 2, Papers in Analysis, 91-95.

Schmidli, H., 2008. Stochastic Control in Insurance. Springer-Verlag, London.

Seal, H.L., 1969. Stochastic Theory of a Risk Business. Wiley, New York.

Shubik, M., Thompson, G.L., 1959. Games of economic survival, Naval Research Logistics Quarterly 6, 111-123.

Takeuchi, K., 1962. A remark on economic survival game, Journal of the Operations Research Society of Japan 4, 114-121.

Yuen, K. C., Wang, G., Li, W. K., 2007. The Gerber-Shiu expected discounted penalty function for risk processes with interest and a constant dividend barrier. Insurance: Mathematics and Economics 40, 104-112. 